\title{
Extensions of the Inner Automorphism Group of a Factor
}

By

\author{
Marie CHODA*
}

\section{Introduction}

Let $M$ be the crossed product $R(G, A, \alpha)$ of a von Neumann algebra $A$ by a locally compact group $G$ under a continuous action $\alpha$. By $\operatorname{Aut}(M, A)$ we shall denote the group of all automorphisms of $M$, each of which is an extension of an automorphism of $A$. A systematic attempt to study $\operatorname{Aut}(M, A)$ for a finite factor $M$ by the group measure space construction has been made in [11]. For the crossed product $M$ of a von Neumann algebra $A$ by a discrete countable group $G$ of freely acting automorphisms of $A$, some results concerning the structure of an elment of $\operatorname{Aut}(M, A)$, which is inner on $M$, have been obtained in [2], [3], [8] and [9], and generalized in [1]. Some relations between elements in $\operatorname{Aut}(M, A)$ and two-cocycles on $G$ have been studied for a general crossed product of a von Neumann algebra $A$ by a discrete countable group, or a locally compact group $G$ under an action ([6], [10], [12], [14]).

In this paper, we consider this generalized crossed product in the form $M=R(G, A, \alpha, v)$ of a factor $A$ by a locally compact group $G$ under an action $\alpha$ with a factor set $\{v(g, h) ; g, h \in G\}$ (cf. Definition in below). In $\S 2$, we shall study the structure of the normal subgroup $K$ of $\operatorname{Aut}(M, A)$, each element of which acts on $A$ as an inner automorphism. Under a certain condition, the group $K$ is isomorphic to the direct product of $\operatorname{Int}(A)$ and $\chi(G)$, where $\operatorname{Int}(A)$ is the group of natural extensions $\operatorname{Ad} u(u \in A)$ and $\chi(G)$ is the character

* Communicated by H. Araki, October 31, 1977.

Department of Mathematics, Osaka Kyoiku University, Osaka 543, Japan. 
group of $G$ (Theorem 1 and Corollary 4). In $\S 3$, we shall restrict our interest to a discrete countable group $G$ and study the structure of the normal subgroup $\operatorname{Int}(M, A)$ of $\operatorname{Aut}(M, A)$, each element of which is an inner automorphism of $M$. If the action under $\alpha$ of all elements in $G$ except the identity is outer on $A$, then $\operatorname{Int}(M, A)$ is isomorphic to an extension group of $\operatorname{Int}(A)$ by $G$ (Theorem 7).

\section{Extensions of Inner Automorphisms}

Let $A$ be a von Neumann algebra acting on a separable Hilbert space $H$. By $\operatorname{Aut}(A)$ we shall denote the group of all automorphisms (*-preserving) of $A$ and by $\operatorname{Int}(A)$ the group of all inner automorphisms of $A$. For a locally compact group $G$, we denote by $K(H ; G)$ the vector space of all continuous $H$-valued functions on $G$ with compact support. Considering the inner product in $K(H ; G)$ defined by

$$
(\xi, \eta)=\int_{G}(\xi(g), \eta(g)) d g, \quad \xi, \eta \in K(H ; G),
$$

$K(H ; G)$ is a pre Hilbert space, where $d g$ is a fixed left Haar measure of $G$. The completion of $K(H ; G)$ with respect to this inner product is denoted by $L^{2}(H ; G)$. A map $\alpha$ of $G$ into $\operatorname{Aut}(A)$ is called an action of $G$ on $A$, if for each fixed $a$ in $A$, the map: $g \in G \rightarrow \alpha_{\mathrm{g}}(a) \in A$ is $\sigma$-strongly * continuous and $\alpha$ satisfies the following condition (1);

$$
i(g, h)=\alpha_{g h}^{-1} \alpha_{g} \alpha_{h} \in \operatorname{Int}(A), \quad g, h \in G .
$$

For such a map $\alpha$, a family $\{v(g, h) ; g, h \in G\}$ of unitaries in $A$ is called a factor set associated with the action $\alpha$, if the map $:(g, h) \in G$ $\times G \rightarrow v(g, h) \in A$ is $\sigma$-strongly * continuous and the following conditions (2) and (3) are satisfied;

$$
\begin{aligned}
& i(g, h)=A d v(g, h), \quad g, h \in G, \\
& v(g, h k) v(h, k)=v(g h, k) \alpha_{k}^{-1}(v(g, h)), \quad g, h, k \in G,
\end{aligned}
$$

where $\operatorname{Ad} u$ is an automorphism of $A$ such that $\operatorname{Ad} u(a)=u a u^{*}$ for $a$ in $A$. In the sequel, we assume that $\alpha_{1}=\iota$, where 1 is the identity 
of $G$ and $\iota$ is the identity automorphism of $A$. On the Hilbert space $L^{2}(H ; G)$, we shall denote by $\pi_{\alpha}$ the representation of $A$ such that

$$
\left(\pi_{\alpha}(a) \xi\right)(h)=\alpha_{h}^{-1}(a) \xi(h), \quad h \in G, \xi \in L^{2}(H ; G) .
$$

By $\rho$, we shall denote a map of $G$ into the unitary group on $L^{2}(H ; G)$ such that

$$
(\rho(g) \xi)(h)=v\left(g, g^{-1} h\right) \xi\left(g^{-1} h\right), \quad h \in G, \xi \in L^{2}(H ; G) .
$$

By the direct computation, we have that

$$
\rho(g) \rho(h)=\rho(g h) \pi_{a}(v(g, h)), \quad g, h \in G
$$

and $\pi_{\alpha}$ and $\rho$ satisfiy the covariance relation;

$$
\rho(g) \pi_{\alpha}(a) \rho(g)^{*}=\pi_{\alpha}\left(\alpha_{g}(a)\right), \quad g \in G, a \in A .
$$

The von Neumann algebra on $L^{2}(H ; G)$ generated by $\pi_{\alpha}(A)$ and $\rho(G)$ is called the crossed product of $A$ by $G$ with the factor set $\{v(g, h) ; g, h \in G\}$ respect to $\alpha$ and denoted by $R(G, A, \alpha, v)$. If the action $\alpha$ is a representation of $G$ into $\operatorname{Aut}(A)$ and the factor set $\{v(g, h) ; g, h \in G\}$ associated with the action $\alpha$ is the trivial set, that is, $v(g, h)$ is the identity for every $g, h$ in $G$, then $R(G, A, \alpha, v)$ is the usual crossed product ([16]), which we shall denote by $R(G, A, \alpha)$.

At first, we shall be concerned with the group of all extensions to $R(G, A, \alpha, v)$ of the inner automorphism group of a factor. Fix a von Neumann algebra $A$ equipped with an action $\alpha$ of a locally compact group $G$ and a factor set $\{v(g, h) ; g, h \in G\}$ associated with the action $\alpha$. Throughout this paper, we shall denote by $M$ the crossed product $R(G, A, \alpha, v)$. By $\operatorname{Aut}(M, A)$, we shall denote the group of automorphisms of $M$ sending $\pi_{\alpha}(A)$ onto itself :

$$
\operatorname{Aut}(M, A)=\left\{\beta \in \operatorname{Aut}(M) ; \beta\left(\pi_{\alpha}(A)\right)=\pi_{\alpha}(A)\right\} \text {. }
$$

It is clear that all inner automorphisms of $A$ admit natural extensions $\operatorname{Ad} u(u \in u(A))$ to $M$ and the automorphisms $\alpha_{G}$ admit natural liftings $\operatorname{Ad} \rho(g)(g \in G)$, where $u(A)$ is the group of unitaries in $A$. By the same notation $\operatorname{Int}(A)$ and $\alpha(G)$ we shall denote the set of such automorphisms of $M$ : 


$$
\operatorname{Int}(A)=\left\{\operatorname{Ad} u \in \operatorname{Aut}(M) ; u \in u\left(\pi_{\alpha}(A)\right)\right\} .
$$

Let $K$ be the group of all extensions to $M$ of the inner automorphism group of $A$ :

$$
K=\left\{\beta \in \operatorname{Aut}(M, A) ; \beta \text { is inner on } \pi_{\alpha}(A)\right\} .
$$

Theorem 1. Let $A$ be a factor equipped with an action $\alpha$ of $a$ locally compact group $G$ and a factor set $\{v(g, h) ; g, h \in G\}$ associated with the action $\alpha$. If $\alpha$ is such that $\pi_{\alpha}(A)^{\prime} \cap M$ is the scalar multiples of the identity, then $K$ is isomorphic to the direct product of $\operatorname{Int}(A)$ and $\chi(G)$, where $\chi(G)$ is the group of all continuous characters of $G$.

Proof. Take a $\beta$ in $K$. Let $u$ be a unitary in $\pi_{\alpha}(A)$ such that $\beta(a)=u a u^{*}$ for all $a$ in $\pi_{\alpha}(A)$. Then, for each $a$ in $\pi_{\alpha}(A)$ and $g$ in $G$, we have that

$$
\begin{aligned}
u \rho(g) a \rho(g)^{*} u^{*} & =\beta\left(\rho(g) a \rho(g)^{*}\right)=\beta(\rho(g)) \beta(a) \beta(\rho(g))^{*} \\
& =\beta(\rho(g)) u a u^{*} \beta(\rho(g))^{*},
\end{aligned}
$$

so that $\rho(g)^{*} u^{*} \beta(\rho(g)) u$ is contained in $\pi_{\alpha}(A)^{\prime} \cap M$. Since $\pi_{\alpha}(A)^{\prime} \cap M$ is the scalar multiples of the identity $I$, we have a $\chi$ in $\chi(G)$ such that

$$
\beta(\rho(g))=\chi(g) u \rho(g) u^{*} .
$$

In fact, put $\chi(g) I=\rho(g) * u^{*} \beta(\rho(g)) u$, then we have that

$$
\begin{aligned}
\chi(g h) I & =\rho(g h)^{*} u^{*} \beta(\rho(g h)) u=\rho(g h)^{*} u^{*} \beta\left(\rho(g) \rho(h) \pi_{\alpha}(v(g, h))\right) u \\
& =\rho(g h)^{*} u^{*} \beta(\rho(g)) \beta(\rho(h)) u \pi_{\alpha}(v(g, h)) \\
& =\chi(g) \rho(g h)^{*} \rho(g) u^{*} \beta(\rho(h)) u \pi_{\alpha}(v(g, h)) \\
& =\chi(g) \chi(h) I .
\end{aligned}
$$

For each character $\chi$ of $G$, put

$$
(u(\chi) \xi)(g) \overline{=\chi(g)} \xi(g), \quad g \in G, \xi \in L^{2}(H ; G),
$$

where $\overline{\chi(g)}$ is the complex conjugate of $\chi(g)$. Then $u(\chi)$ is a unitary satisfying

$$
u(\chi) a u(\chi)^{*}=a, \quad a \in \pi_{\alpha}(A),
$$


and

$$
u(\chi)(\rho(g)) u(\chi)^{*}=\overline{\chi(g)} \rho(g), \quad g \in G .
$$

Let $\delta(\chi)$ be an automorphism of $M$ induced by $u(\chi)$, then $\delta(\chi)$ belongs to the group $K$.

For a $\beta$ in $K$, let $u$ be a unitary in $\pi_{\alpha}(A)$ such that $\beta(a)=u a u^{*}$ for all $a$ in $\pi_{\alpha}(A)$. Take a $\chi$ in $\chi(G)$ satisfying the property (8), then we have that

$$
(\beta \delta(\chi))(a)=\beta(a)=\operatorname{Ad} u(a), \quad a \in \pi_{\alpha}(A)
$$

and

$$
(\beta \delta(\chi))(\rho(g))=\overline{\chi(g)} \beta(\rho(g))=\operatorname{Ad} u(\rho(g)), \quad g \in G,
$$

so that $\beta \cdot \delta(\chi)$ belongs to $\operatorname{Int}(A)$. Thus every $\beta$ in $K$ has a form $\beta=\operatorname{Ad} u \cdot \delta(\chi)$ for some $u$ in $\pi_{\alpha}(A)$ and $\chi$ in $\chi(G)$. Such a decomposition is unique. In fact, if

$$
\operatorname{Ad} u \cdot \delta(\chi)=\operatorname{Ad} w \cdot \delta\left(\chi^{\prime}\right), \quad u, w \in \pi_{\alpha}(A), \chi, \chi^{\prime} \in \chi(G),
$$

then we have that on $\pi_{\alpha}(A), \operatorname{Ad} w^{*} u$ is the identity automorphism. Since $A$ is a factor, it follows that $w$ is a scalar multiple of $u$, which implies that $\operatorname{Ad} u=\operatorname{Ad} w$ on $M$ and that $\delta(\chi)=\delta\left(\chi^{\prime}\right)$.

By the property (10), we have that, for $\chi$ and $\chi^{\prime}$ in $\chi(G)$, $\delta(\chi)=\delta\left(\chi^{\prime}\right)$ if and only if $\chi=\chi^{\prime}$.

Therefore, defining a map $\sigma$ of the direct product of $\operatorname{Int}(A)$ and $\chi(G)$ onto $K$ by $\sigma(\gamma, \chi)=\gamma \cdot \delta(\chi),(\gamma \in \operatorname{Int}(A), \chi \in \chi(G))$, we have an isomorphism of $K$ onto the direct product of $\operatorname{Int}(A)$ and $\chi(G)$.

Let $K_{0}$ be the group of all extensions to $M$ of the identity automorphism of $A$ :

$$
K_{0}=\left\{\beta \in \operatorname{Aut}(M, A) ; \beta \text { is the identity on } \pi_{\alpha}(A)\right\} .
$$

Corollary 2. Let $A, \alpha, G$ and $\{v(g, h) ; g, h \in G\}$ be as in Theorem 1. The group $K_{0}$ is isomorphic to $\chi(G)$.

Denote by $[G, G]$ the commutator group of $G$, that is, $[G, G]$ 
is the closed group generated by $\left\{g h g^{-1} h^{-1} ; g, h \in G\right\}$. A group $G$ is called perfect if $[G, G]$ coincides with $G$.

Corollary 3. Let $A, G, \alpha$ and $\{v(g, h) ; g, h \in G\}$ be as in Theorem 1. The following three statements are equivalent:

(a) $K$ coincides with $\operatorname{Int}(A)$;

(b) $K_{0}$ is the trivial group $\{c\}$;

(c) $G$ is perfect.

Proof. By Theorem 1 and Corollary 2, it is clear that the statements (a) and (b) are equivalent and that they are equivalent to the condition that $\chi(G)=\{1\}$. On the other hand, $\chi(G)$ is the group $\operatorname{Hom}(G, T)$ of all continuous homomorphism of $G$ into $T$, where $T$ is the unit circle of the complex plane. Since $T$ is an abelian group, it follows that for each $\chi$ in $\chi(G),[G, G]$ is contained in the kernel of $\chi$. Hence $\chi(G)$ is isomorphic to $\operatorname{Hom}(G /[G, G], T)$. Thus the condition that $\chi(G)=\{1\}$ is equivalent to $G=[G, G]$, which is statement (c).

Especially, assume that $G$ is a discrete countable group. If $\alpha_{g}$ is an outer automorphism of $A$ for all $g$ in $G$ except the unit, then by [5, Corollary 3], we have that $\pi_{\alpha}(A)^{\prime} \cap M$ is the scalar multiples of the identity. Therefore, we have the following corollary:

Corollary 4. Let $A$ be a factor equipped with an action $\alpha$ of a discrete countable group $G$ and a factor set $\{v(g, h) ; g, h \in G\}$ associated with the action $\alpha$. Assume that $\alpha_{\mathrm{g}}$ is an outer automorphism of $A$ for all $g$ in $G$ except the unit. Then $K$ is isomorphic to the direct product of $\operatorname{Int}(A)$ and $\chi(G)$, so that $K_{0}$ is isomorphic to $\chi(G)$. The three statements in Corollary 3 are equivalent.

\section{Extensions as Inner Automorphisms.}

In this section, we shall be concerned with extensions of automorphisms of $A$ to $M$ which are inner on $M$. 
Throughout this section, we shall treat a factor equipped with an action of a discrete countable group $G$ and a factor set $\{v(g, h)$; $g, h \in G\}$ associated with the action $\alpha$. For $M=R(G, A, \alpha, v)$, we shall denote by $\operatorname{Int}(M, A)$ the group of inner automorphisms of $M$ sending $\pi_{\alpha}(A)$ into iteself and by $u(M, A)$ the group of unitaries in $M$ normalizing $\pi_{\alpha}(A)$ :

$$
\operatorname{Int}(M, A)=\left\{\beta \in \operatorname{Int}(M) ; \beta\left(\pi_{\alpha}(A)\right)=\pi_{\alpha}(A)\right\},
$$

and

$$
u(M, A)=\left\{u \in u(M) ; u \pi_{\alpha}(A) u^{*}=\pi_{\alpha}(A)\right\} .
$$

We shall determine a relation among $\operatorname{Int}(M, A), \operatorname{Int}(A)$ and $G$.

Theorem 5. Let $A$ be a factor equipped with an action $\alpha$ of a discrete countable group $G$ and a factor set $\{v(g, h) ; g, h \in G\}$ associated with the action $\alpha$. Then each $u$ in $u(M, A)$ has a form;

$$
u=w w^{\prime} \rho(g), \quad w \in u\left(\pi_{\alpha}(A)\right), w^{\prime} \in u\left(\pi_{\alpha}(A)^{\prime} \cap M\right), g \in G .
$$

By the same technique as [8; Corollary 1] or [9; Theorem], we can prove this theorem. For the sake of completeness, we shall give a proof of Theorem 5 .

Proof. Take a $u$ in $u(M, A)$. Let

$$
u=\sum_{g \in G} a(g) \rho(g) \quad a(g) \in \pi_{\alpha}(A) \text {, (in the } \sigma \text {-strong topology) }
$$

be the Fourier expansion of $u([5 ;$ Lemma 1$])$. By the property that $u \pi_{\alpha}(A) u^{*}=\pi_{\alpha}(A)$, we have that

$$
\sum_{g \in G} \alpha(g) \alpha_{g}(a) \rho(g)=\sum_{g \in G} u a u^{*} a(g) \rho(g), \quad a \in \pi_{\alpha}(A),
$$

so that

$$
a(g) \alpha_{g}(a)=u a u^{*} a(g), \quad a \in \pi_{\alpha}(A), g \in G .
$$

If $\alpha_{g}^{-1} \mathrm{Ad} u$ is an outer automorphism of $\pi_{\alpha}(A)$, then we have that $a(g)=0$. Since $u$ is unitary, it follows that there exists a $g$ in $G$ such that $\alpha_{g}^{-1} \mathrm{Ad} u$ is inner on $\pi_{\alpha}(A)$. Let $w$ be a unitary in $\pi_{\alpha}(A)$ such that on $\pi_{\alpha}(A), \alpha_{g}^{-1} \mathrm{Ad} u=\mathrm{Ad} w$. Put $w^{\prime}=\rho(g)^{*} u w^{*}$, then $w^{\prime}$ belongs to $\pi_{\alpha}(A)^{\prime} \cap M$. 
Corollary 6. Let $A, G, \alpha$ and $\{v(g, h) ; g, h \in G\}$ be as in Theorem 5. Each $\beta$ in $\operatorname{Int}(M, A) \cap \operatorname{Aut}(A)$ has a form:

$$
\beta=\gamma \alpha_{g}, \quad \gamma \in \operatorname{Int}(A), g \in G \text {. }
$$

Theorem 7. Let $A, G, \alpha$ and $\{v(g, h) ; g, h \in G\}$ be as in Theorem 5. Assume that $\alpha_{g}$ is an outer automorphism of $A$ for all $g$ in $G$ except the identity. Then $u(M, A)$ is isomorphic to an extension group of $u(A)$ by $G$ and $\operatorname{Int}(M, A)$ is isomorphic to an extension group of $\operatorname{Int}(A)$ by $G$. If $M$ is the usual crossed product $R(G, A, \alpha)$, then these extensions are a semi-direct product.

Proof. If $\alpha_{g}$ is an outer automorphism of $A$ for all $g$ in $G$ except the unit, then $\pi_{\alpha}(A)^{\prime} \cap M$ is the scalar multiples of the identity ([5; Corollary 3]). Hence, by Theorem 5, each $u$ in $u(M, A)$ has a form :

$$
u=w \rho(g), \quad w \in u\left(\pi_{\alpha}(A)\right), g \in G .
$$

If

$$
w \rho(g)=w^{\prime} \rho(h), \quad w, w^{\prime} \in u\left(\pi_{\alpha}(A)\right), g, h \in G,
$$

then we have that

$$
\begin{aligned}
w^{\prime *} w & =\rho(h) \rho(g)^{*}=\rho(h) \rho\left(g^{-1}\right) \pi_{\alpha}\left(v\left(g, g^{-1}\right)^{*}\right) \\
& =\rho\left(h g^{-1}\right) \pi_{\alpha}\left(v\left(h, g^{-1}\right) v\left(g, g^{-1}\right)^{*}\right) .
\end{aligned}
$$

On the other hand, by [4; Theorem 6], there exists a faithful normal expectation $e$ of $M$ onto $\pi_{\alpha}(A)$ such that $e(\rho(g))=0$ for all $g$ in $G$ except the unit. Therefore, if the relation (16) is satisfied for $g$ and $h$ in $G$ such that $g \neq h$, then we have that $w^{\prime *} w=0$ by (17), which is a contradiction. Thus the decomposition of $u$ in $u(M, A)$ with the form (15) is unique. We shall define a map $\sigma$ on the set $u\left(\pi_{\alpha}(A)\right) \times G$ by $\sigma(w, g)=w \rho(g), \quad w \in u\left(\pi_{\alpha}(A)\right), g \in G$. Define a multiplication on $u\left(\pi_{\alpha}(A)\right) \times G$ by

$$
(w, g)\left(w^{\prime}, h\right)=\left(w \alpha_{g}\left(w^{\prime}\right) \alpha_{g h}\left(\pi_{\alpha}(v(g, h))\right), g h\right),
$$

then $\sigma$ is an isomorphism of the extension group $E\left(G, u\left(\pi_{\alpha}(A)\right), \alpha, v\right)$ of $u\left(\pi_{\alpha}(A)\right)$ by $G$ under the multiplication (18) onto $u(M, A)$. If 
$M$ is the usual crossed product $R(G, A, \alpha)$, then we may always take $v(g, h)=I$ for all $g, h$ in $G$, so that mapping $\sigma$ gives an isomorphism of a semi direct product of $u\left(\pi_{\alpha}(A)\right)$ by $G$ onto $u(M, A)$.

Similarly, define a multiplication in the set $\operatorname{Int}(A) \times G$ by

$$
(\operatorname{Ad} u, g)(\operatorname{Ad} w, h)=\left(\operatorname{Ad}\left(u \alpha_{g}(w) \alpha_{g h}\left(\pi_{\alpha}(v(g, h))\right), g h\right) .\right.
$$

The group $\operatorname{Int}(A)$ is isomorphic to the factor group $u\left(\pi_{\alpha}(A)\right) / T I$ of $u\left(\pi_{\alpha}(A)\right)$ by the normal subgroup $\{\mu I ; \mu \in T\}$. The extension group $E(G, \operatorname{Int}(A), \alpha, v)$ of $\operatorname{Int}(A)$ by $G$ under the multiplication (19) is isomorphic to the factor group $E\left(G, u\left(\pi_{\alpha}(A)\right), \alpha, v\right) / T I \times\{1\}$ of $E\left(G, u\left(\pi_{\alpha}(A)\right), \alpha, v\right)$ by the normal subgroup $T I \times 1=\{(\mu I, 1) ; \mu \in T\}$. On the other hand, $E\left(G, u\left(\pi_{\alpha}(A)\right), \alpha, v\right) / T I \times 1$ is isomorphic to the factor group $u(M, A) / T I$ of $u(M, A)$ by the normal subgroup $T I$, which is isomorphic to $\operatorname{Int}(M, A)$. Thus $\operatorname{Int}(M, A)$ is isomorphic to the extension group $E(G, \operatorname{Int}(A), \alpha, v)$ of $\operatorname{Int}(A)$ by $G$ under the multiplication (19).

\section{References}

[1] Behencke, H., Automorphisms of crossed product Tohoku, Math.J., 21 (1969), 580-600.

[2] Choda, H. and Choda, M., On extensions of automorphisms of abelian von Neumann algebras, Proc.Japan Acad., 43 (1967), 295-299.

[3] Choda, H., On freely acting automorphisms of operator algebras, Kodai Math. Sem. Report, 26 (1974), 1-21.

[ 4] Choda, M., Some relations of $\mathrm{II}_{1}$-factors on free groups, Math. Japonicae, 22 (1977) 383-394.

[5] Choda, M., A characterization of crossed products of factors by discrete outer automorphism groups, To appear in J. Math. Soc. Japan, 31.

[6] Feldman, J., and Moore, C. C., Ergodic equivalence relations, cohomology and von Neumann algebras, II, Preprint.

[7] Hewitt, E. and Ross, K. A., Abstract Harmonic Analysis, I, Springer-Verlag.

[8] Haga, Y. and Takeda, Z., Correspondence between subgroups and subalgebras in a cross product von Neumann algebra, Tohoku Math.J., 19 (1967), 315-323.

[9] Nakamura, M. and Takeda, Z., On inner automorphisms of certain finite factors, Proc. Japan Acad., 3 J (1961), 31-32.

[10] Nakamura, M. and Takeda, Z., On extensions of finite factors, I, Proc.Japan Acad., $\mathbf{3 5}$ (1959), 149-154.

[11] Singer, I. M., Automorphisms of finite factors, Amer.J.Math., 17 (1955), 117-183.

[12] Sutherland, C. E., Cohomology and extensions of von Neumann algebras, I, Preprint.

[13] Takesaki, M., Duality for crossed products and the structure of von Neumann algebras of type III, Acta Math., 131 (1974) 249-310.

[14] Zeller-Meier, G., Produits croisés d'une $\mathrm{C}^{*}$-algèbra par un groupe d'automorphisms, J.Math.Pures et Appl., 47 (1968), 101-239. 
